\title{
The Computational Complexity of the Local Postage Stamp Problem
}

\author{
Jeffrey Shallit* \\ Department of Computer Science \\ University of Waterloo \\ Waterloo, Ontario, Canada N2L 3G1 \\ shallit@graceland.uwaterloo.ca
}

October 31, 2018

\begin{abstract}
The well-studied local postage stamp problem (LPSP) is the following: given a positive integer $k$, a set of positive integers $1=a_{1}<a_{2}<\cdots<a_{k}$ and an integer $h \geq 1$, what is the smallest positive integer which cannot be represented as a linear combination $\sum_{1 \leq i \leq k} x_{i} a_{i}$ where $\sum_{1 \leq i \leq k} x_{i} \leq h$ and each $x_{i}$ is a non-negative integer? In this note we prove that LPSP is NP-hard under Turing reductions, but can be solved in polynomial time if $k$ is fixed.
\end{abstract}

\section{Introduction}

The local postage-stamp problem, or LPSP for short, can be informally defined as follows. One is given a supply of stamps of $k$ different denominations, $1=a_{1}<a_{2}<\cdots<a_{k}$, and an envelope that has room for at most $h$ different stamps. What is the smallest amount of postage $N_{h}\left(a_{1}, a_{2}, \ldots, a_{k}\right)$ that cannot fit on the envelope? For example, if the available denominations are $1 \phi, 4 \phi, 7 \phi$, and $8 \phi$, and the envelope has room for 3 stamps, then all amounts of postage $\leq 24$ can be provided but 25 c cannot. Hence $N_{3}(1,4,7,8)=25$.

A more formal statement of LPSP is given in the abstract.

In this note we consider the computational complexity of LPSP. If $N=\sum_{1 \leq i \leq k} x_{i} a_{i}$, we call $\left(x_{1}, x_{2}, \ldots, x_{k}\right)$ a representation for $N$ and $\sum_{1 \leq i \leq k} x_{i}$ the weight of the representation. If further $\sum_{1 \leq i \leq k} x_{i}$ is minimum among all representations for $N$, we call $\left(x_{1}, x_{2}, \ldots, x_{k}\right)$ a minimum-weight representation for $N$. If the denominations $a_{i}$ and bound $h$ are given in unary, then a simple dynamic programming algorithm can determine the minimum-weight representation for all integers $N \leq h a_{k}+1$ in polynomial-time, and hence we can compute

${ }^{*}$ Currently on sabbatical (until July 2002) at Department of Computer Science, University of Arizona, P. O. Box 210077, Tucson, AZ 85721-0077, USA. 
$N_{h}\left(a_{1}, a_{2}, \ldots, a_{k}\right)$ in polynomial time. We therefore assume for the rest of this paper that all inputs are provided in binary.

LPSP was apparently introduced by Rohrbach [7, 8] in 1937, and since then dozens of papers have been written about it and a variant, the global postage-stamp problem (GPSP); see Guy [3, pp. 123-127]) for a brief survey. Despite this, no general results on the computational complexity of the problem seem to be known up to now; for example, Alter and Barnett [1] asked if $N_{h}\left(a_{1}, \ldots, a_{k}\right)$ "can be expressed by a simple formula". Selmer [9] discussed efficient algorithms for the case where $k \leq 3$.

In the next section we prove that LPSP is NP-hard under Turing reductions, and in Section 3 we give a polynomial-time algorithm for LPSP when $k$ is fixed.

\section{LPSP is NP-hard}

We prove that LPSP is NP-hard by reducing from a related problem, the Frobenius problem (see, for example, Guy [3, pp. 113-114]). In the Frobenius problem, we are given an integer $k \geq 1$ and $k$ positive integers $a_{1}, a_{2}, \ldots, a_{k}$ with $\operatorname{gcd}\left(a_{1}, a_{2}, \ldots, a_{k}\right)=1$, and we are asked to compute $g\left(a_{1}, a_{2}, \ldots, a_{k}\right)$, the largest integer which cannot be expressed as a non-negative

integer linear combination $\sum_{1 \leq i \leq k} x_{i} a_{i}$. The Frobenius problem is well-studied, but it was only fairly recently that it was proved NP-hard (under Turing reductions) by RamírezAlfonsín [6].

Before we give the reduction, we need a technical lemma.

Lemma 1 Let $1=a_{1}<a_{2}<\cdots<a_{k}$. Define

$$
h_{0}=\sum_{1 \leq i \leq k}\left\lfloor\frac{a_{i+1}}{a_{i}}\right\rfloor,
$$

and

$$
h_{1}=h_{0}+\left\lceil\frac{\left(h_{0}+1\right) a_{k-1}}{a_{k}-a_{k-1}}\right\rceil .
$$

Then

(a) $N_{h_{0}}\left(a_{1}, a_{2}, \ldots, a_{k}\right)>a_{k}$;

(b) $N_{h_{0}+i}\left(a_{1}, a_{2}, \ldots, a_{k}\right)>(i+1) a_{k}$ for all $i \geq 0$;

(c) $N_{h}\left(a_{1}, a_{2}, \ldots, a_{k}\right)>(h+1) a_{k-1}-a_{k}$ for all $h \geq h_{1}$.

(d) $N_{h+1}\left(a_{1}, a_{2}, \ldots, a_{k}\right)=N_{h}\left(a_{1}, a_{2}, \ldots, a_{k}\right)+a_{k}$ for all $h \geq h_{1}$.

(e) There exists a constant $c \geq-1$ such that $h a_{k}-N_{h}\left(a_{1}, a_{2}, \ldots, a_{k}\right)=c$ for all $h \geq h_{1}$.

(f) If $h \geq h_{1}$, then $N_{h}\left(a_{1}, a_{2}, \ldots, a_{k}\right)=h a_{k}-g\left(a_{k}-a_{k-1}, a_{k}-a_{k-2}, \ldots, a_{k}-a_{1}, a_{k}\right)$, where $g$ is the Frobenius number.

Remark. Parts (a)-(f) can be essentially found more or less verbatim in the paper of Selmer [9]; the only difference in our presentation is that we explicitly compute the bounds $h_{0}, h_{1}$. 
Proof. (a) Consider finding a representation $n=\sum_{1 \leq i \leq k} x_{i} a_{i}$ for an integer $n, 0 \leq n \leq$ $a_{k}$, using the greedy algorithm. We use at most $\left\lfloor a_{k} / \bar{a}_{k-1}\right\rfloor$ copies of $a_{k-1}$, then at most $\left\lfloor a_{k-1} / a_{k-2}\right\rfloor$ copies of $a_{k-2}$, etc. The choice of $h_{0}$ thus allows us to form the greedy representation of all such $n$.

(b) We prove this by induction on $i$. For $i=0$ the result is just part (a). Otherwise, suppose $N_{h_{0}+i}\left(a_{1}, a_{2}, \ldots, a_{k}\right)>(i+1) a_{k}$. Then every integer $n, 0 \leq n \leq(i+1) a_{k}$ has a representation of weight $\leq h_{0}+i$ and by adding a single copy of $a_{k}$, we can represent every integer $m, 0 \leq m \leq(i+2) a_{k}$ with weight $\leq h_{0}+i+1$.

(c) Set $i=j+\left\lceil\frac{\left(h_{0}+1\right) a_{k-1}}{a_{k}-a_{k-1}}\right\rceil, j \geq 0$, and apply (b).

(d) The numbers that have representations of weight $\leq h+1$ can be divided into two not necessarily disjoint subsets:

$$
\begin{aligned}
& S_{1}=\left\{n: \text { some representation of } n \text { of weight } \leq h+1 \text { has } x_{k}>0\right\} \\
& S_{2}=\left\{n: \text { some representation of } n \text { of weight } \leq h+1 \text { has } x_{k}=0\right\} .
\end{aligned}
$$

Now every element of $S_{1}$ can be written as $a_{k}+t$, where $t$ has a representation of weight $\leq h$. It follows that

$$
\left\{a_{k}, a_{k}+1, a_{k}+2, \ldots, N_{h}\left(a_{1}, a_{2}, \ldots, a_{k}\right)+a_{k}-1\right\} \subseteq S_{1},
$$

but $N_{h}\left(a_{1}, h_{2}, \ldots, a_{k}\right)+a_{k} \notin S_{1}$. On the other hand, the numbers in $S_{2}$ have representations of weight $\leq h+1$ using just the numbers $\left\{a_{1}, a_{2}, \ldots, a_{k-1}\right\}$, and so the largest element of $S_{2}$ is $\leq(h+1) a_{k-1}$. Furthermore, by (a) and the fact that $h_{1} \geq h_{0}$, we have $\left\{0,1, \ldots, a_{k}\right\} \subseteq S_{2}$. It follows that provided

$$
N_{h}\left(a_{1}, a_{2}, \ldots, a_{k}\right)+a_{k}>(h+1) a_{k-1},
$$

we have $N_{h+1}\left(a_{1}, a_{2}, \ldots, a_{k}\right)=N_{h}\left(a_{1}, a_{2}, \ldots, a_{k}\right)+a_{k}$. But (11) follows from (c).

(e) Using (d), a simple induction gives $N_{h_{1}+i}\left(a_{1}, a_{2}, \ldots, a_{k}\right)=N_{h_{1}}\left(a_{1}, a_{2}, \ldots, a_{k}\right)+i a_{k}$ for all $i \geq 0$. Then $\left(h_{1}+i\right) a_{k}-N_{h_{1}+i}\left(a_{1}, a_{2}, \ldots, a_{k}\right)=h_{1} a_{k}-N_{h_{1}}\left(a_{1}, a_{2}, \ldots, a_{k}\right)$ for all $i \geq 0$; so we may take $c=h_{1} a_{k}-N h_{1}\left(a_{1}, a_{2}, \ldots, a_{k}\right)$. Since $N_{h_{1}}\left(a_{1}, a_{2}, \ldots, a_{k}\right) \leq h_{1} a_{k}+1$, it follows that $c \geq-1$.

(f) Suppose $h \geq h_{1}$. Then by (e) we have $h a_{k}-N_{h}\left(a_{1}, a_{2}, \ldots, a_{k}\right)$ is independent of $h$. Now $h a_{k}-t$ has a representation of weight $h^{\prime} \leq h$ iff

$$
\begin{aligned}
t & =h a_{k}-\sum_{1 \leq i \leq k} x_{i} a_{i} \\
& =\left(\sum_{1 \leq i \leq k-1} x_{i}\left(a_{k}-a_{i}\right)\right)+\left(h-h^{\prime}\right) a_{k},
\end{aligned}
$$

i.e., if $t$ has a representation of any weight using the basis $a_{k}-a_{k-1}, a_{k}-a_{k-2}, \ldots, a_{k}-a_{1}, a_{k}$, since we can choose $h$ to be arbitrarily large. But the largest $t$ with no representation in the basis $a_{k}-a_{k-1}, a_{k}-a_{k-2}, \ldots, a_{k}-a_{1}, a_{k}$ is just the Frobenius number

$$
g\left(a_{k}-a_{k-1}, a_{k}-a_{k-2}, \ldots, a_{k}-a_{1}, a_{k}\right) .
$$

(Since $a_{1}=1$, we have $\operatorname{gcd}\left(a_{k}-a_{k-1}, a_{k}-a_{k-2}, \ldots, a_{k}-a_{1}, a_{k}\right)=1$.) It follows that $h a_{k}-N_{h}\left(a_{1}, a_{2}, \ldots, a_{k}\right)=g\left(a_{k}-a_{k-1}, a_{k}-a_{k-2}, \ldots, a_{k}-a_{1}, a_{k}\right)$. 
Theorem 2 Given positive integers $b_{1}<b_{2}<\cdots<b_{k}$ with $\operatorname{gcd}\left(b_{1}, b_{2}, \ldots, b_{k}\right)=1$, we can determine in polynomial time integers $h, a_{1}=1, a_{2}, \ldots, a_{k}, a_{k+1}, a_{k+2}$ such that $g\left(b_{1}, b_{2}, \ldots, b_{k}\right)=h a_{k+2}-N_{h}\left(a_{1}, a_{2}, \ldots, a_{k}, a_{k+1}, a_{k+2}\right)$.

Proof. By a theorem of Brauer [2, Corollary to Thm. 1], we know that $g\left(b_{1}, b_{2}, \ldots, b_{k}\right)<$ $b_{k} b_{1}$. Define $b_{k+1}=b_{k} b_{1}$ and $b_{k+2}=b_{k} b_{1}+1$. Then clearly $g\left(b_{1}, b_{2}, \ldots, b_{k}\right)=g\left(b_{1}, b_{2}, \ldots, b_{k}, b_{k+1}, b_{k+2}\right)$. Now we have, by Lemma 1 (f), that

$$
h_{1} a_{k+2}-N_{h_{1}}\left(b_{k+2}-b_{k+1}, b_{k+2}-b_{k}, \ldots, b_{k+2}-b_{1}, b_{k+2}\right)=g\left(b_{1}, b_{2}, \ldots, b_{k}, b_{k+1}, b_{k+2}\right),
$$

where

$$
h_{1}=h_{0}+\left\lceil\frac{\left(h_{0}+1\right) a_{k+1}}{a_{k+2}-a_{k+1}}\right\rceil,
$$

and

$$
h_{0}=\sum_{1 \leq i \leq k+2}\left\lfloor\frac{a_{i+1}}{a_{i}}\right\rfloor .
$$

Note that $b_{k+2}-b_{k+1}=1$.

Since the Frobenius problem reduces to LPSP, and the Frobenius problem is NP-hard, so is LPSP.

\section{A polynomial-time algorithm for fixed $k$}

I observe that results of Kannan [4 5 provide a polynomial-time algorithm for the local postage-stamp problem for any fixed dimension $k$. Let $Q$ be a given copolyhedron (an intersection of a finite number of half-spaces, possibly closed, possibly open) in $\mathbb{R}^{p+l}$. Let $A$ be an $m \times n$ matrix, $B$ be a $m \times p$ matrix, and $C$ be a column vector of dimension $m$, all with integer entries. Kannan proved that assertions of the form

$$
\text { " } \forall y \in Q / \mathbb{Z}^{l} \exists x \in \mathbb{Z}^{n} \text { such that } A x+B y \leq C \text { " }
$$

can be tested in polynomial time when $l+n+p$ is fixed. (Here $Q / \mathbb{Z}^{l}=\left\{y \in \mathbb{R}^{p}\right.$ : there exists $t \in \mathbb{Z}^{l}$ such that $[y, t] \in Q$.) Thus if $k$ is fixed, by taking $n=k, l=p=1$, and $Q=\{[y, y]: 0 \leq y \leq M-1\}$, we can decide in polynomial time whether $\forall t, 0 \leq t \leq$ $M-1, \exists x_{1}, x_{2}, \ldots, x_{k} \in \mathbb{Z}$ such that $x_{1}, x_{2}, \ldots, x_{k} \geq 0, \sum_{1 \leq i \leq k} x_{i} a_{i}=t$, and $\sum_{1 \leq i \leq k} x_{i} \leq h$. Now we use a binary search on $M$ to find the largest $M$ for which the statement holds. This gives us $N_{h}\left(a_{1}, a_{2}, \ldots, a_{k}\right)$. Note that $Q$ is the intersection of four half-spaces. Kannan's algorithm is quite complicated and this method is likely not to be useful in practice.

\section{References}

[1] R. Alter and J. A. Barnett. A postage stamp problem. Amer. Math. Monthly 87 (1980), 206-210.

[2] A. Brauer. On a problem of partitions. Amer. J. Math. 64 (1942), 299-312.

[3] R. K. Guy. Unsolved Problems in Number Theory. Springer-Verlag, 2nd edition, 1994. 
[4] R. Kannan. Solution of the Frobenius problem. Technical Report CMU-CS-89-204, Carnegie-Mellon University, Dept. of Computer Science, November 1989. Extended abstract in C. E. Veni Madhavan, ed., Proc. 9th Conf. Found. Software Tech. Theor. Comput. Sci., Lecture Notes in Computer Science, Vol. 405, pp. 242-251.

[5] R. Kannan. Test sets for integer programs, $\forall \exists$ sentences. In Polyhedral Combinatorics, Vol. 1 of DIMACS Series in Discrete Mathematics and Theoretical Computer Science, pp. 39-47. Amer. Math. Soc., 1990.

[6] J. L. Ramírez-Alfonsín. Complexity of the Frobenius problem. Combinatorica 16 (1996), 143-147.

[7] H. Rohrbach. Ein Beitrag zur additiven Zahlentheorie. Math. Zeitschrift 42 (1937), 1-30.

[8] H. Rohrbach. Anwendung eines Satzes der additiven Zahlentheorie auf eine gruppentheoretische Frage. Math. Zeitschrift 42 (1937), 538-542.

[9] E. S. Selmer. On the postage stamp problem with three stamp denominations. Math. Scand. 47 (1980), 29-71. 